\title{
ON RADEMACHER'S EXTENSION OF THE GOLDBACH-VINOGRADOFF THEOREM
}

\author{
BY \\ RAYMOND AYOUB
}

1. Introduction. The Goldbach problem for odd numbers $n$ seeks to prove that the equation

$$
n=p_{1}+p_{2}+p_{3}
$$

where the $p_{i}$ are prime numbers, is always solvable. Hardy and Littlewood, using the now classical "circle" method, proved that if $B(n)$ is the number of solutions of (1), then under certain assumptions on the zeros of Dirichlet $L$-functions,

$$
B(n)=\varsigma^{\prime}(n) \frac{n^{2}}{2(\log n)^{3}}+o\left(\frac{n^{2}}{(\log n)^{3}}\right)
$$

where $\widetilde{S}^{\prime}(n)$, the so-called "singular series," was proved to be greater than 0 for all odd $n$.

Rademacher [1], using simplifications of his own as well as of Landau, proved under similar assumptions that if $k$ be a positive integer, $a_{i}$ $(i=1,2,3)$ integers with $\left(a_{i}, k\right)=1$, and $A(n)$ the number of solutions of (1) with the restriction that the primes $p_{i}$ belong to the residue classes $a_{i}$ modulo $k$, then $\left({ }^{1}\right)$

$$
A(n)=\subseteq(n) I(n)+o\left(\frac{n^{2}}{(\log n)^{3}}\right)
$$

where

$$
I(n)=\iint \frac{d u d v}{\log u \log v \log (n-u-v)},
$$

and the range of integration is defined by the inequalities $u \geqq 2, v \geqq 2, u+v$ $\leqq n-2$. He further proved that $S(n)>0$ provided certain necessary arithmetic restrictions on $n$ hold. It may be shown that

$$
I(n)=\frac{n^{2}}{2(\log n)^{3}}+o\left(\frac{n^{2}}{(\log n)^{3}}\right) .
$$

Received by the editors July 9, 1952.

(1) Rademacher considers the more general problem of the number of representations of $n$ as a sum of $s(s \geqq 3)$ primes belonging to preassigned residue classes modulo $k$, and obtains a precise error term. 
Vinogradoff [2], using the theorems of his own on estimates of trigonometric sums together with a theorem on the uniformity of distribution of primes in an arithmetic progression due to Siegel and Walfisz, proved the Hardy-Littlewood result without assumptions.

Following the work of Vinogradoff and Rademacher, we prove the result of Rademacher without recourse to Dirichlet $L$-functions and the assumptions on the location of their zeros.

2. Notations and preliminary results. Let $n$ be an integer chosen sufficiently large, $\nu=\log n, \theta$ is a real number with $|\theta|<1, m$ is any constant $>3$. $f(x) \ll g(x)$ means $f(x)=O(g(x))$, and denote $e^{(2 \pi i / q) x}$ by $e_{q}(x)$.

Theorem 2.1 (Siegel-Walfisz). If $\pi(n, q, t)$ denotes the number of primes $\leqq n$ in the progression $q x+t,(q, t)=1$, and if $0<q \leqq \nu^{3 m}$, then,

$$
\pi(n, q, t)=(1 / \phi(q)) \int_{2}^{n} \frac{d x}{\log x}+O\left((\phi(q))^{-1} n \nu^{-15 m-1}\right),
$$

where the constant implied by the $O$ depends only on $m$.

Theorem 2.2. Let $(h, q)=1, d \mid q,(a, d)=1$, and denote by $(u)$ the set of integers satisfying the conditions $1 \leqq u \leqq q, u \equiv a(\bmod d),(u, q)=1$. If

$$
S(q)=\sum_{(u)} e_{q}(h u),
$$

then,

$$
S(q)=\left\{\begin{array}{l}
\mu(q / d) e_{d}(b a h) \text { if }((q / d), d)=1 \quad \text { and } \quad(q / d) b \equiv 1(\bmod k), \\
0 \quad \text { if }((q / d), d)>1 .
\end{array}\right.
$$

Proof. The proof follows Rademacher.

Let $T(q)=\sum e_{q}(v)$, summed over those $v$ such that $1 \leqq v \leqq q, v \equiv a(\bmod d)$; then

$$
T(q)=\sum_{c \mid q} \sum_{w} e_{q}\left(h \frac{q}{c} w\right)=\sum_{c \mid q} U(c),
$$

where, for given $c, w$ in the inner sum ranges over those integers satisfying $(c, w)=1,1 \leqq w \leqq q, w q / c \equiv a(\bmod d)$. Moreover, if $q_{1} \mid q$ and $T\left(q_{1}\right)=\sum_{t} e_{q_{1}}(h t)$, where $t$ ranges over the set $1 \leqq t \leqq q, t\left(q / q_{1}\right) \equiv a(\bmod d)$, then

$$
T(q)=\sum_{c \mid q_{1}} U(c) .
$$

From (4) and (5), however, we get

$$
\sum_{q_{1} \mid q} \mu\left(q / q_{1}\right) T\left(q_{1}\right)=\sum_{q_{1} \mid q} U\left(q_{1}\right) \sum_{q_{2} \mid q / q_{1}} \mu\left(q / q_{1} q_{2}\right)=U(q)=S(q) .
$$

If $\left(\left(q / q_{1}\right), d\right)=1$, then $T\left(q_{1}\right)=0$; moreover $T\left(q_{1}\right)=e_{q_{1}}(h d) T\left(q_{1}\right)$. Conse- 
quently $T\left(q_{1}\right)=0$ for $q_{1} \nmid h d$. On the other hand for $q_{1} \mid h d,\left(\left(q / q_{1}\right), d\right)=1$, determine $b$ in such a way that $b q / q_{1} \equiv 1(\bmod d)$. Then

$$
T\left(q_{1}\right)=\sum_{1 \leqq v \leqq q_{1} / d} e_{q_{1}}(h(v d+b a))=\left(q_{1} / d\right) e_{q_{1}}(b a h) .
$$

Since $q_{1} \mid d, T\left(q_{1}\right)=0$ for $q_{1}<d$, and the theorem is proved.

Suppose now that

$$
S(x)=S_{i}(x)=\sum_{(p)} e(x p),
$$

where $(p)$ denotes the set of primes satisfying the conditions $p \leqq n$, $p \equiv a(\bmod k)$ with $a=a_{1}, a_{2}, a_{3}$, and $e_{1}(x)=e(x)$, then

$$
A(n)=\int_{0}^{1} S_{1}(x) S_{2}(x) S_{3}(x) e(-n x) d x=\int_{0}^{1} f(x) d x \quad \text { (say). }
$$

As usual, we divide the unit interval into Farey "arcs." Let $h / q=r$ be a rational point of the unit interval with $(h, q)=1$ and $1 \leqq q \leqq \nu^{3 m}$. The major "arc" $B_{r}$ belonging to $r$ is the set of points $x$ in $(0,1)$ with

$$
|x-r| \leqq n^{-1} \nu^{3 m}=\tau^{-1} \text {. }
$$

It is proved that no two major arcs intersect, and if $E$ denotes the set of points not belonging to any $B_{r}$, then $x$ in $E$ has the form

$$
x=h / q+\theta / q \tau \text { with } \nu^{3 m}<q \leqq \tau .
$$

Since $f(x)$ has period 1 , (8) can be written as

$$
A(n)=\sum_{r} \int_{B_{r}} f(x) d x+\int_{E} f(x) d x .
$$

\section{Estimate on the major arcs.}

Theorem 3.1. Let $d=(k, q)$; then if $x$ belongs to $B_{r}$,

$$
S(x)=\frac{1}{\phi(k)} \frac{\mu(q / d)}{\phi(q / d)} e_{d}(h a b) \int_{2}^{n} \frac{e(x z)}{\log x} d x+O\left(n \nu^{-6 m-1}\right) .
$$

Proof. With Vinogradoff, we divide $S(x)$ into $O\left(\nu^{9 m}\right)$ sums of the form

$$
S_{u}(x)=\sum_{u<p \leqq v} e((h / q+z) p),
$$

the range of summation being further restricted by the condition $p$ $\equiv a(\bmod k), 0<v-u \leqq n \nu^{q_{m}}$, and since $x$ is a point of $B_{r}$, it has the form $x=h / q+z$, with $|z| \leqq \tau^{-1}$. We write (11) in the form

$$
S_{u}(x)=\sum_{j} \sum_{u<p \leqq v} e((h / q+z) p),
$$


where $p$ in the inner sum is further restricted by $p \equiv a(\bmod k)$ and $p$ $\equiv j(\bmod q)$. We deduce by the Chinese remainder theorem

$$
S_{u}(x)=\sum_{j \equiv a(\bmod d)} \sum_{u<p \leqq v} e((h / q+z) p),
$$

where in the inner sum $p \equiv s(\bmod k q / d)$. Denote the inner sum by $S_{u}^{j}(x)$. Since $p \equiv j(\bmod q)$, we get $e((h / q+z) p)=e(h j / q+u z)+O\left(|z| n \nu^{-9 m}\right)$. On the other hand,

$$
S_{u}^{j}(x)=\left\{e(h j / q) e(u z)+O\left(|z| n \nu^{-9 m}\right)\right\} \sum_{p} 1,
$$

with $p$ satisfying the conditions of the above inner sum. Using Theorem 2.1, we deduce

$$
S_{u}^{j}(x)=(\phi(k q / d))^{-1} e(h j / q) e(u z) I_{1}+O\left((\phi(k q / d))^{-1}\left(n \nu^{-1-15 m}+I_{1}|z| \nu^{-9 m}\right)\right),
$$

where

$$
I_{1}=\int_{u}^{v} \frac{d x}{\log x}
$$

Since $|z||x-u| \leqq|z| n \nu^{-9 m}$, we get

$$
I_{1} e(u z)=\int_{u}^{v} \frac{e(x z)}{\log x} d x+O\left(I_{1}|z| n \nu^{-9 m}\right) .
$$

Consequently,

$$
S_{u}^{j}(x)=(\phi(k q / d))^{-1} \int_{u}^{v} \frac{e(x z)}{\log x} d x+O\left((\phi(k q / d))^{-1}\left(n \nu^{-1-15 m}+I_{1}|z| \nu^{-9 m}\right)\right) .
$$

Summing over $j$, we get,

$$
\begin{aligned}
S_{u}(x)= & (\phi(k q / d))^{-1} \int_{u}^{v} \frac{e(x z)}{\log x} d x \sum_{j} e(h j / q) \\
& +O\left((\phi(k q / d))^{-1}\left(n \nu^{-1-15 m}+I_{1}|z| n \nu^{-9 m}\right)\right) \sum_{j} 1 .
\end{aligned}
$$

Here $j$ ranges over the set $1 \leqq j \leqq q,(j, q)=1, j \equiv a(\bmod d)$. If $((q / d), d)=1$, and $b$ is determined such that $(q / d) b \equiv 1(\bmod d)$, we get, by Theorem 2.3,

$$
\begin{aligned}
S_{u}(x)= & (\phi(k q / d))^{-1} \mu(q / d) e(h b a / d) \int_{u}^{v} \frac{e(x z)}{\log x} d x \\
& +O\left(\left(\phi(q / d)(\phi(k q / d))^{-1}\right)\left(n \nu^{-1-15 m}+I_{1}|z| n \nu^{-9 m}\right)\right),
\end{aligned}
$$

while if $((q / d), d)>1$, 


$$
S_{u}(x)=O\left(\left(\phi(q / d)(\phi(k q / d))^{-1}\right)\left(n \nu^{-1-15 m}+I_{1}|z| n \nu^{-9 m}\right)\right) .
$$

Continuing with (12), we observe that $1=((q / d), d)=((q / d), \quad(q, k))$ $=((q / d), k)$. Hence

$$
\begin{aligned}
S_{u}(x)= & (\phi(k))^{-1}(\phi(q / d))^{-1} \mu(q / d) e(h a b / d) \int_{u}^{v} \frac{e(x z)}{\log x} d x \\
& +O\left(n \nu^{-1-15 m}+I_{1}|z| n \nu^{-9 m}\right) .
\end{aligned}
$$

Summing over all intervals, we get

$$
\begin{aligned}
S(x)= & \left.(\phi(k))^{-1} \phi(q / d)\right)^{-1} \mu(q / d) e(h a b / d) \int_{2}^{n} \frac{e(x z)}{\log x} d x \\
& +O\left(n \nu^{-15 m-1} \nu^{9 m}+|z| n \nu^{-9 m} \int_{2}^{n} \frac{d x}{\log x}\right) .
\end{aligned}
$$

Since

$$
|z| n \nu^{-9 m} \int_{2}^{n} \frac{d x}{\log x}=O\left(n \nu^{-6 m-1}\right)
$$

the result follows.

THEOREM 3.2.

$$
\begin{aligned}
\int_{B r} f(x) d x= & \frac{1}{\phi(k)^{3}} \frac{\mu(q / d)}{\phi(q / d)^{3}} e\left(\left(h b\left(a_{1}+a_{2}+a_{3}\right) / d\right)-n h / q\right) \\
& \cdot \int_{-r^{-1}}^{r-1}\left(I_{2}(z)\right)^{3} e(-n z) d z+O\left(\frac{1}{\phi(q / d)^{2}} n^{2} \nu^{-6 m-3}\right),
\end{aligned}
$$

where

$$
I_{2}(z)=\left\{{ }_{2}^{n} \frac{e(x z)}{\log x} d x .\right.
$$

Proof. An easy calculation shows that $I_{2}(z)=O(\zeta)$ where

$$
\zeta= \begin{cases}n \nu^{-1} & \text { if } \quad|z| \leqq n^{-1}, \\ |z|^{-1} \nu^{-1} & \text { if } \quad n^{-1}<z \leqq n \nu^{-3 m} .\end{cases}
$$

We have

$$
(\phi(k))^{-1}(\phi(q / d))^{-1} \mu(q / d) e(h a b / d) I_{2}(z)=O\left((\phi(q / d))^{-1} I_{2}(z)\right),
$$

and since $\phi(q / d) n \nu^{-6 m-1}\left(I_{2}(z)\right)^{-1}=O(1)$, we deduce

$$
\begin{aligned}
S_{1}(x) S_{2}(x) S_{3}(x)= & (\phi(k))^{-3}(\phi(q / d))^{-3} \mu(q / d) e\left(h b\left(a_{1}+a_{2}+a_{3}\right) / d\right) \\
& \cdot\left(I_{2}(z)\right)^{3}+O\left(\phi(q / d)^{2} n \nu^{-6 m-1}\left(I_{2}(z)\right)^{2}\right) .
\end{aligned}
$$


Therefore

$$
\begin{aligned}
\int_{B r} f(x) d x & =\int_{-r^{-1}}^{r^{-1}} S_{1}(x) S_{2}(x) S_{3}(x) e(-(h / q+z) n) d z \\
& =\left(\phi(k)^{-3}(\phi(q / d))^{-3} \mu(q / d) e\left(h b\left(a_{1}+a_{2}+a_{3}\right) / d\right) I+I_{3}(z),\right.
\end{aligned}
$$

where

$$
\begin{gathered}
I=\int_{-\tau^{-1}}^{\tau^{-1}}\left(I_{2}(z)\right)^{3} e(-n z) d z, \\
\left.I_{3}(z)=O\left((\phi(q / d))^{-2} n \nu^{-6 m-1}\right) \int_{0}^{n^{-1} \nu^{3 m}} \zeta^{2} d z\right) \\
=O\left((\phi(q / d))^{-2} n \nu^{-6 m-1} \int_{0}^{n^{-1}} n^{2} \nu^{-2} d z+\int_{n^{-1}}^{n^{-1} \nu^{2} m} n^{-2} z^{-2} d z\right) \\
=O\left(\left(\phi(q / d)^{-2} n^{2} \nu^{-6 m-3}\right)\right) .
\end{gathered}
$$

\section{Corollary.}

$$
\begin{aligned}
\sum_{r} \int_{B_{r}} f(x) d x= & \frac{1}{\phi(k)^{3}} \sum_{q} \frac{\mu(q / d)}{\phi(q / d)^{3}} \sum_{h} e\left(b h\left(a_{1}+a_{2}+a_{3}\right) / d-h n / q\right) I \\
& +O\left(n^{2} \nu^{-3}{ }^{m-3}\right) .
\end{aligned}
$$

Here the inner sum ranges over the set $i \leqq h \leqq q,(h, q)=1$, and the outer sum over the set $(d,(q / d))=1, q \leqq \nu^{3 m}$.

\section{Estimate on the minor arc.}

THEOREM 4.1. Let $(u)$ and $(v)$ be two increasing sequences of positive integers and $w$ a positive integer. Let $1<N^{\prime}<N_{1}, n_{1}=\log N_{1}, 1<U_{0}<U_{1} \leqq N_{1}$, $1<\tau<N_{1}, x=h / q+\theta / q \tau, \delta=(w, q), q=\delta q_{1}, w=\delta w_{1}$, and

$$
T=\sum_{u} \sum_{v} e(x w u v)
$$

where $u$ runs through the elements of the sequence $(u)$ satisfying the inequalities $U_{0}<u \leqq U_{1}$ and, for given $u, v$ ranges over those elements of the sequence $(v)$ satisfying the inequalities $N^{\prime} / u<v \leqq N_{1} / u$; then

$$
T=O\left(N_{1}\left(n_{1} / U_{0}+U_{1} / N_{1}+q_{1} n_{1}^{3} / N_{1}+n_{1}^{2} / q_{1}+w_{1} n_{1}^{2} / \tau\right)^{1 / 2}\right) .
$$

Proof. The proof may be found in Vinogradoff [2].

Denote by $H$ the product of all primes $\leqq n^{1 / 2}$, and by $(d)$ the sequence of integers satisfying the condition $d \mid H, d \leqq n$. Using a reasoning similar to that used in the proof of Theorem 2.2, we derive the following expression for $S(x)$ 


$$
S(x)=\sum_{(d)} \mu(d) S_{d}+O\left(n^{1 / 2}\right),
$$

where

$$
S_{d}=\sum_{u} e(x d u) .
$$

Here $u$ ranges over the sequence satisfying the conditions $d u \leqq n, d u$ $\equiv a(\bmod k)$. We have

$$
\sum_{(d)} \mu(d) S_{d}=\sum_{\left(d_{0}\right)} S_{d}-\sum_{\left(d_{1}\right)} S_{d}=S_{0}-S_{1} \quad \text { (say), }
$$

where $\left(d_{0}\right)$ is the sequence of elements of $(d)$ having an even number of divisors and $\left(d_{1}\right)$ those elements of $(d)$ having an odd number of divisors. We estimate $S_{0} ; S_{1}$ can be estimated in exactly the same way. Write $\lambda=\nu^{2(m+1)}$, and divide $S_{0}$ into three sums,

$$
S_{0}=\sum_{d \leqq \lambda} S_{d}+\sum_{\lambda<d \leqq n \lambda_{-1}} S_{d}+\sum_{n \lambda-1 \leqq d \leqq n} S_{d}=T_{1}+T_{2}+T_{3} .
$$

It is understood of course that the index $d$ ranges over the set $\left(d_{0}\right)$ satisfying the given inequalities.

To estimate $T_{1}$, we observe that if $d^{\prime}=d /(k, d)$ and $a^{\prime}$ is a solution of the congruence $d x \equiv a(\bmod k)$ and $n_{2}=n(k, d) / k d$, then

$$
S_{d}=\sum_{u \leqq n_{2}} e\left(x d^{\prime}\left(k u+a^{\prime}\right)\right) .
$$

Consequently, $\left|S_{d}\right| \leqq q$; it follows that

$$
T_{1} \ll n \nu^{-m+2} .
$$

To estimate $T_{2}$, we apply Theorem 4.1 . We have

$$
T=\sum_{d} \sum_{u} e(x d u)
$$

with the prescribed ranges of summation. We have here $N_{1}=n, U_{0}=\lambda$, $U_{1}=n \lambda^{-1}, w=1$. Theorem 4.1 yields

$$
T_{2} \ll n\left(\nu^{-2 m-1}\right)^{1 / 2} \ll n \nu^{-m+2} .
$$

We turn now to the estimate of $T_{3}$. We have

$$
T_{3}=\sum_{d} \sum_{u} e(x d u)
$$

summed over the prescribed ranges for $d$ and $u$. Interchange the order summation, then

$$
T_{3}=\sum_{u \leqq \lambda} \sum_{n \lambda^{-1}<d \leqq n / u} e(x d u)=\sum_{u} T(u)
$$


with the inner sum further restricted by the condition $d u \equiv a(\bmod k)$. We divide the sequence $(d)$ into two sequences $\left(d^{\prime}\right)$ and $\left(d^{\prime \prime}\right)$ where $\left(d^{\prime}\right)$ is the set of $(d)$ having all prime divisors $\leqq \nu^{3 m}$ and $\left(d^{\prime \prime}\right)$ those elements of $(d)$ having at least one prime divisor $>\nu^{3 m}$. $\left(d_{0}\right)$ is then divided into two corresponding sets $\left(d_{0}^{\prime}\right)$ and $\left(d_{0}^{\prime \prime}\right)$. We get $T(u)=T^{\prime}(u)+T^{\prime \prime}(u)$ where the right-hand summands correspond to the sets $\left(d_{0}^{\prime}\right)$ and $\left(d_{0}^{\prime \prime}\right)$ respectively. We estimate now the number of terms $D$ of the set $\left(d^{\prime}\right)$ which satisfy the conditions $d \leqq n / u$ and $1 \leqq u \leqq \lambda$. To this end suppose that an element $d$ of $\left(d^{\prime}\right)$ have $j$ prime divisors. Then $\left(\nu^{3 m}\right)^{i} \geqq n \lambda^{-1}$, and hence if $n$ be chosen sufficiently large $j>\nu / 6 m \log \nu$. If then $\tau(d)$ be the number of divisors of $d$, we get

$$
\tau(d)=2^{j}>2^{\nu / 6 m \log \nu}>n^{1 / 9 m \log \nu},
$$

and since

$$
\sum_{1 \leqq v \leqq n_{1}} \tau(v) \ll n_{1}(\nu+1),
$$

where $n_{1}=n / u$, we conclude that

$$
D n^{1 / 9 m \log \nu} \ll n_{1}(\nu+1) \ll n_{1} n^{1 / 9 m \log \nu} n^{-1 / 9 m \log \nu} \nu^{m} \nu^{-m}(\nu+1) .
$$

Therefore $D \ll n_{1} \nu^{-m}$. Consequently we deduce that

$$
T(u)=T^{\prime \prime}(u)+O\left(\nu^{-m} n u^{-1}\right) .
$$

For the sum $T^{\prime \prime}(u)$ we have evidently $j<\nu$, hence

$$
T^{\prime \prime}(u)=\sum_{j} T_{j}(u)
$$

where $T_{j}(u)$ is summed over those $d$ belonging to $\left(d_{0}^{\prime \prime}\right)$ satisfying the inequalities $n \lambda^{-1}<d \leqq n_{1}$, and having exactly $j$ prime divisors $>\nu^{3 m}$. In order to estimate the sum $T_{j}(u)$ we consider with Vinogradoff the more general sum

$$
T_{j}{ }^{\prime}(u)=\sum_{v} \sum_{w} e(x u v w)
$$

where $v$ ranges over all primes $>\nu^{3 m}$ belonging to $(d)$ and, for given $v$, $w$ ranges over those numbers satisfying the inequalities $n \lambda^{-1} / v<w \leqq n_{1} / v$, the congruence $u v w \equiv a(\bmod k)$ and containing exactly $j-1$ prime divisors $>\nu^{3 m}$ and belonging to $\left(d_{1}\right)$. Every term $e(x d u)$ of the sum $T_{j}(u)$ is found in the sum $T_{j}^{\prime}(u)$ and indeed is found exactly $j$ times. In addition, however, $T_{j}^{\prime}(u)$ contains terms of the form $e\left(x p^{2} w_{1}\right)$ with $n \lambda^{-1} / p^{2}<w_{1} \leqq n_{1} / p^{2}$, where $p>\nu^{3 m}$, and $w_{1}$ runs over elements of $\left(d_{0}\right)$ containing $j-2$ prime divisors $>\nu^{3 m}$. These terms evidently occur without duplication. For given $p$, the number of $w_{1} p^{2}$ satisfying $n \lambda^{-1} / p^{2}<w_{1} \leqq n_{1} / p^{2}$ is $\leqq n_{1} / p^{2}$, consequently

$$
T_{j}^{\prime}(u)=j T_{j}(u)+O\left(\sum_{\nu^{3 m}<p \leqq n^{1 / 2}} n_{1} / p^{2}\right)=j T_{j}(u)+O\left(\nu^{-3 m} n u^{-1}\right) .
$$


We now apply Theorem 4.1 to the sum $T_{j}^{\prime}(u)$. We take $U_{0}=\nu^{3 m}, U_{1}=n^{1 / 2}$, $N^{\prime}=n \lambda^{-1}$, and conclude

$$
T(u) \ll n / u\left(u \nu^{-3 m+2}\right)^{1 / 2} \ll n \nu^{-3 m / 2+1} u^{-1 / 2} .
$$

Therefore, $T_{j}(u) \ll j^{-1} n u^{-1 / 2} \nu^{-3 m / 2+1}$, from which we deduce that

$$
T^{\prime \prime}(u) \ll n u^{-1 / 2} \nu^{-3 m / 2+1} \log \nu,
$$

and hence that

$$
T(u) \ll n u^{-1 / 2} \nu^{-3 m / 2+1} \log \nu+n u^{-1} \nu^{-m} .
$$

Summing over $u$, we deduce that

$$
T_{3} \ll n \nu^{-m+1} \log \nu+n \nu^{-m} \log \nu \ll n \nu^{-m+2} .
$$

Using (19), (20), (21), (22), and (23), we conclude the following:

Theorem 4.2. Let $m$ be any constant $>3$,

$$
x=h / q+\theta / q \tau, \quad(h, q)=1, \quad \nu^{3 m}<q \leqq \tau, \quad \tau=n \nu^{-3 m} ;
$$

then

$$
\sum_{(p)} e(x p)=O\left(n \nu^{-m+2}\right)
$$

5. The asymptotic formula and proof of the theorem.

TheOREM 5.1. If $m>9 / 4$, then

$$
I=\int_{-\tau^{-1}}^{\tau^{-1}}\left(I_{2}(z)\right)^{3} e(-n z) d z=\frac{n^{2}}{2 \nu^{3}}+O\left(\frac{n^{2}}{\nu^{7 / 2}} \log \nu\right) .
$$

Proof. Vinogradoff [2].

Using this result, we deduce readily that

$$
\sum_{q}(\phi(q / d))^{-3} \mu(q / d) \sum_{h} e\left(b h\left(a_{1}+a_{2}+a_{3}\right) / d-h n / q\right) I=O\left(n^{2} \nu^{-4}\right),
$$

where the inner sum ranges over the set of $h$ such that $1 \leqq h \leqq q,(h, q)=1$, and the outer sum over those $q$ satisfying $((q / d), d)=1, q>\nu^{3 m}$. This result, together with (17), permits us to conclude that

$$
\sum_{r} \int_{B_{r}} f(x) d x=S(n) \frac{n}{2 \nu^{3}}+O\left(n^{2} \nu^{-7 / 2} \log \nu\right),
$$

where $\mathfrak{S}(n)$, the singular series, is given by

$$
\Im(n)=\frac{1}{\phi(k)^{3}} \sum_{q=1}^{\infty} \frac{\mu(a / d)}{\phi(q / d)^{3}} \sum_{1 \leqq h \leqq q} e\left(h b\left(a_{1}+a_{2}+a_{3}\right) / d-h n / q\right),
$$

where as above $q$ is restricted by $((q / d), d)=1$ and $h$ by $(h, q)=1$. On the 
other hand, using Theorem 4.2 , we get

$$
\begin{aligned}
\int_{E} f(x) d x & \ll \int_{E}\left|S_{1}(x) S_{2}(x) S_{3}(x)\right| d x \\
& \ll n \nu^{-m+2} \int_{0}^{1}\left|S_{2}(x) S_{3}(x)\right| d x \\
& \ll n \nu^{-m+2}\left(\int_{0}^{1}\left|S_{2}(x)\right|^{2} d x\right)^{1 / 2}\left(\int_{0}^{1}\left|S_{3}(x)\right|^{2} d x\right)^{1 / 2} \\
& \ll n \nu^{-m+2} n \nu^{-1} \ll n^{2} \nu^{-m+1} .
\end{aligned}
$$

From (9), (26), and (28), we conclude

$$
A(n)=\Im(n) \frac{n^{2}}{2(\log n)^{3}}+O\left(\frac{n^{2}}{(\log n)^{7 / 2}} \log \log n\right) .
$$

On the other hand Rademacher has shown that if $n$ is odd and $n \equiv a_{1}+a_{2}$ $+a_{3}(\bmod k)$, then

$$
\Im(n)=\frac{C}{k^{2}} \prod_{p \mid k} \frac{p^{3}}{(p-1)^{3}+1} \prod_{p \mid n, p \nmid k} \frac{(p-1)\left((p-1)^{2}-1\right)}{(p-1)^{3}+1} \prod_{p>2}\left(1+\frac{1}{(p-1)^{3}}\right),
$$

where throughout $p>2, C=2$ for odd $k$, and $C=8$ for even $k$. If $n$ fails to satisfy the above conditions, then $\mathfrak{S}(n)=0$.

We formulate the:

MaIn Theorem. Let $k$ be a positive integer, $a_{1}, a_{2}, a_{3}$ be residue classes modulo $k$ with $\left(a_{i}, k\right)=1$. If $n$ is a sufficiently large odd integer satisfying the congruence $n \equiv a_{1}+a_{2}+a_{3}(\bmod k)$, then $n$ can be represented as a sum of three primes belonging respectively to the residue classes $a_{1}, a_{2}, a_{3}$, modulo $k$. The asymptotic formula for the number of representations is given by (29).

6. Concluding remarks. The method of Linnik-Tchudakoff will provide another proof of this result. The corresponding question for the simultaneous Goldbach-Waring problem may be posed and solved.

\section{BIBLIOGRAPHY}

1. H. A. Rademacher, Ueber eine Erweiterung des Goldbachschen Problems, Math. Zeit. vol. 25 (1926).

2. I. Vinogradoff, Some theorems concerning the theory of primes, Matematcheskii Sbornik vol. 2 (44) (1937).

\section{HARVARD UNIVERSITY,}

Cambridge, Mass. 